SLAC-PUB-8135

March, 1999

\title{
New Developments in the Next Linear Collider Beam Delivery System Design ${ }^{1}$

\author{
P. Tenenbaum, L. Eriksson, T. Markiewicz, T.O. Raubenheimer, A. Ringwall \\ Stanford Linear Accelerator Center, Stanford University, Stanford, CA
}

\begin{abstract}
We review many developments in the design of the Beam Delivery System for the Next Linear Colllider. The collimation system has been redesigned to reduce nonlinearities and ease tolerances, and the IP Switch line has been adjusted to permit a 300 meter longitudinal separation between the two detectors. Magnet designs have been changed to reduce the number of distinct magnet classes, produce a smoother vacuum chamber, and improve pumping. A revised set of interaction-region quadrupoles achieves the optical requirements of the system with improved beam stay-clears, and performance of the final focus at various energies has been simulated and found acceptable. Studies have begun on the set of feedbacks required to maintain the stability of the system and the changes in tolerances thus implied.
\end{abstract}

Contributed to 1999 IEEE Particle Accelerator Conference, New York, New York, March 29 - April 2, 1999

${ }^{1}$ Work supported by the Department of Energy, Contract DE-AC03-76SF00515. 


\title{
NEW DEVELOPMENTS IN THE NEXT LINEAR COLLIDER BEAM DELIVERY SYSTEM DESIGN ${ }^{\dagger}$
}

\author{
$\underline{\text { P. Tenenbaum }}^{\ddagger}$, L. Eriksson, T. Markiewicz, T.O. Raubenheimer, A. Ringwall \\ Stanford Linear Accelerator Center, Stanford University, Stanford, CA
}

\section{Abstract}

We review many developments in the design of the Beam Delivery System for the Next Linear Colllider. The collimation system has been redesigned to reduce nonlinearities and ease tolerances, and the IP Switch line has been adjusted to permit a 300 meter longitudinal separation between the two detectors. Magnet designs have been changed to reduce the number of distinct magnet classes, produce a smoother vacuum chamber, and improve pumping. A revised set of interaction-region quadrupoles achieves the optical requirements of the system with improved beam stay-clears, and performance of the final focus at various energies has been simulated and found acceptable. Studies have begun on the set of feedbacks required to maintain the stability of the system and the changes in tolerances thus implied.

\section{INTRODUCTION}

The Beam Delivery System of the Next Linear Collider (NLC) encompasses all of the beamlines downstream of the main linac. These beamlines include a collimation section which removes particles which would otherwise intersect downstream apertures; an "IP Switch" which deflects the beam towards one of the two interaction points; a 10 milliradian arc for IP separation and muon protection; a section for measurement and correction of $x y$ coupling; a chromatically corrected final focus; and the interaction point and detector. Figure 1 shows a schematic layout of the beam delivery system. Table 1 shows some of the critical system parameters.

Table 1: Parameters of NLC Beam Delivery System as a function of center-of-mass energy. Operation at $120 \mathrm{~Hz}$ and 95 bunches per train is assumed.

\begin{tabular}{|c|c|c|}
\hline Parameter & $500 \mathrm{GeV}$ & $1000 \mathrm{GeV}$ \\
\hline $\begin{array}{l}\text { Bunch } \\
\text { Charge }\end{array}$ & $0.75 \times 10^{10}$ & $0.75 \times 10^{10}$ \\
\hline$\beta_{x, y}, \mathrm{~mm}$ & $10 \times 0.1$ & $10 \times 0.125$ \\
\hline$\gamma \epsilon_{x, y}, \mathrm{~mm} . \mathrm{mrad}$ & $4 \times 0.06$ & $4 \times 0.06$ \\
\hline$\sigma_{z}, \mu \mathrm{m}$ & 90 & 90 \\
\hline $\begin{array}{l}\text { Luminosity, } \\
\mathrm{cm}^{-2} \mathrm{sec}^{-1}\end{array}$ & $7.3 \times 10^{33}$ & $13.5 \times 10^{33}$ \\
\hline
\end{tabular}

\footnotetext{
$\dagger$ Work supported by the Department of Energy, contract DE-AC03$76 \mathrm{SF} 00515$.

‡Email:quarkpt@slac.stanford.edu
}

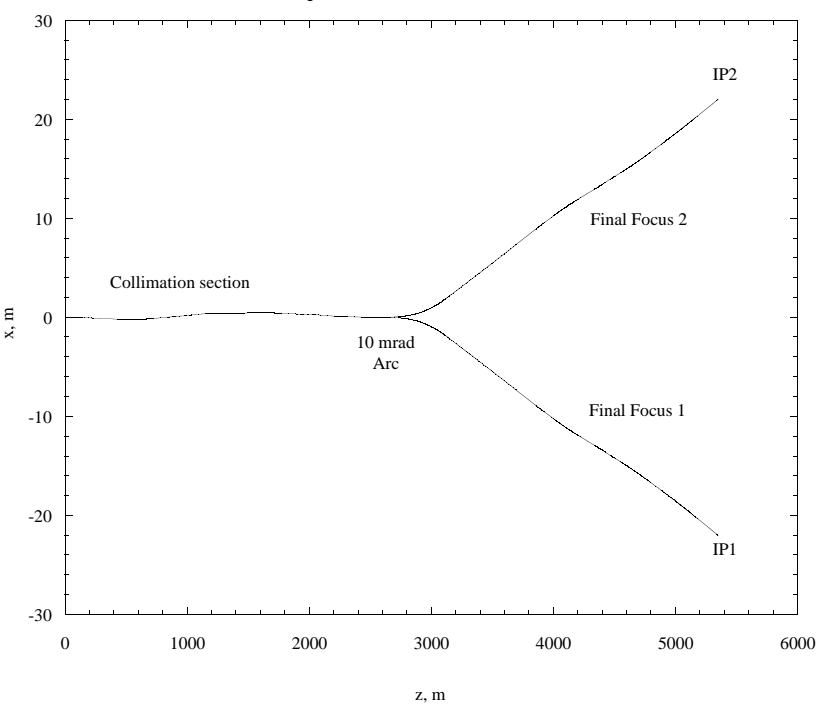

Figure 1: Schematic of NLC Beam Delivery System.

The Beam Delivery System was initially designed during the 1996 NLC study[1], and in the intervening time a number of aspects of the design have been further optimized and revisited. We review these developments below.

\section{MAGNET DESIGNS}

the original BDS design used a large number of different magnet designs, and incorporated arbitrary changes in the magnet apertures. The designs have been regularized to ease buildability. At the present time the BDS uses 5 standard quad geometries, 3 standard bend core geometries, and 3 standard sextupole geometries. All magnets upstream of the final focus have a full aperture of $1.2 \mathrm{~cm}$, while all magnets in the final focus have a full aperture of $3.0 \mathrm{~cm}$ in order to maintain the desired beam stay-clears (14 $\sigma_{x}, 60 \sigma_{y}$ ) for the worst-quality beam at the lowest energy ( $175 \mathrm{GeV}$ per beam, $\left.\gamma \epsilon_{x, y}=5.0 \times 0.14 \mathrm{~mm} . \mathrm{mrad}\right)$. Reduction in the number of distinct magnet families is expected to improve maintenance and reduce construction costs.

Increasing the vacuum chamber to $1.2 \mathrm{~cm}$ O.D. or larger from the original design (which used $1.0 \mathrm{~cm}$ O.D. in many locations) has other positive implications. With full apertures of $1.2 \mathrm{~cm}$ or larger, and assuming a $1 \mathrm{~mm}$ thick vacuum chamber, a base pressure on the order of $5 \times 10^{-8}$ torr can be achieved without taking extraordinary measures in the areas of vacuum chamber processing or vacuum pump spacing. By eliminating most of the aperture transitions in the system, a smoother vacuum system without transitioninduced wakefields can be used. One negative implication 
is that about $10 \%$ of magnets have pole-fields in excess of 7 $\mathrm{kG}$ at $1 \mathrm{TeV}$ C.M., and would need to be replaced in order to run NLC with a center-of-mass energy of $1.5 \mathrm{TeV}$.

\section{COLLIMATION SYSTEM}

The three design constraints which dictated the design of the collimation system were:

- Jitter amplification due to collimator wakefields should be less than $25 \%$

- Collimation system should be capable of removing $1 \%$ of the beam power per degree of freedom $\left(x, x^{\prime}, y, y^{\prime}, \delta\right)$ indefinitely without damage

- Collimation system should be capable of surviving direct impact of a single bunch train $\left(10^{12}\right.$ electrons or positrons at $500 \mathrm{GeV}$ ) without damage.

Of the three, it is the third constraint which had the greatest impact on the system design: surviving the impact of a damped bunch train containing so many kilojoules required that the betatron functions at the collimators be enormous, which in turn required a very long $(>2 \mathrm{~km})$ system. Collimating both phases at high-beta points $\left(\beta_{y}>41 \mathrm{~km}\right)$ had other unpleasant implications: sextupoles were required to correct the induced chromaticity; the $R_{34}$ from the IPphase collimators to the Final Doublet (FD) phase collimators was so large that submicron quad drifts could direct the beam into a collimator; the system length limited the number of iterations of collimation possible; large amplitude particles experienced unacceptable phase migration (from the loosely-collimated IP phase into the tight-apertured FD phase). Furthermore, the design was not yet long enough to survive an impact of a buch train at $750 \mathrm{GeV}$ beam energy. An upgrade to $1.5 \mathrm{TeV}$ C.M. would require lengthening the collimation system into the main linac, which would in turn amplify all the difficulties listed above.

Because the existing design is marginal in so many respects, we have begun to consider a design in which the single-train survival requirement is eliminated. In order to implement such a design, all sources of large oscillations coming from upstream must be sufficiently slow that the machine protection system (MPS) can detect them before they generate a bunch orbit which impacts a collimator. Since the energy of the linac cannot be assured on a pulseto-pulse basis, the new design must extract off-energy particles or bunches before performing betatron collimation, rather than combining $\delta$ and $x$ collimation as the present system does. In the new design, the betatron functions at the collimators can be reduced by over an order of magnitude, with a similar increase in magnet tolerances. The beam is now small enough that DC collimation of the halo can cause collimator damage. To eliminate this risk, the thick halo absorbers are eliminated entirely from the system; instead, a large number of spoilers, each approximately $1 / 4$ radiation length in thickness, causes particles at large amplitudes to be far off energy, and the particles are removed by a dispersive section at the end of the collimation section. The changes in the system permit the collimation to be performed at $45^{\circ}$ intervals in betatron phase, resulting in better collimation (and equal collimation depth for all phases). The new design also has a sufficient bandpass that sextupoles and other high-order multipoles are not needed.

\section{IP SWITCH DESIGN}

The beam delivery system contains an arc which deflects the beam by $10 \mathrm{mrad}$ before entering the final focus system. This provides a separation between the two interaction regions of 40 meters, which is insufficient. The IP Switch module now incorporates an additional module 287 meters in length on the path to one of the two IR's. This provides a separation between IR's which is acceptable.

\section{FINAL FOCUS}

The apertures of the final doublet magnets of the final focus were found to be too small to accomodate beams at lower energy. In addition, the required pole-tip field in the permanent magnet Q1 was unacceptably high, especially since this magnet partially overlaps the detector solenoid's field. A compromise solution was arrived at, in which the lower energies (350-750 $\mathrm{GeV} \mathrm{CM}$ ) use a pair of permanent magnets with a larger aperture and smaller gradient, and the higher energies (750-1000 GeV CM) use a single permanent magnet with a higher gradient and smaller aperture but more acceptable pole-tip fields. Table 2 shows the parameters of the final doublet magnets.

Table 2: Parameters of NLC Final Doublet magnets. Q1B and Q1A are used at lower energies, and are replaced by $\mathrm{Q} 1$ at higher energies. "SC" represents a superconducting magnet, "PM" indicates permanent magnets.

\begin{tabular}{|c|c|c|c|c|}
\hline $\begin{array}{c}\text { Name/ } \\
\text { Polarity }\end{array}$ & $\begin{array}{c}L_{\text {eff }} \\
(\mathrm{m})\end{array}$ & $\begin{array}{c}\text { Full Bore } \\
(\mathrm{cm})\end{array}$ & $\begin{array}{c}B_{0} \\
(\mathrm{kG})\end{array}$ & Type \\
\hline \hline Q2A (F) & 2.0 & 2.0 & 10.0 & Fe elec. \\
\hline Q2B (F) & 2.0 & 2.0 & 10.0 & Fe elec. \\
\hline Q1SC (D) & 1.5 & 2.0 & 30.0 & SC \\
\hline Q1B (D) & 0.5 & 2.1 & 11.6 & PM \\
\hline Q1A (D) & 1.0 & 1.8 & 12.5 & PM \\
\hline Q1 (D) & 2.0 & 1.3 & 12.5 & PM \\
\hline
\end{tabular}

Decreasing the gradient of the final lenses increases their total length, which in turn increases the severity of chromatic aberrations. Thus the bandwidth of the final focus can be degraded by selecting longer, weaker final doublet magnets. The bandwidth was improved by adding a fourth quadrupole to the matching region between the end of the chromatic correction section and the final doublet, thus allowing the phase advance of the matching region to be optimized in each plane for bandpass[2], and tuning 3 sex- 
tupoles at IP images in the chromatic correction sections and final dispersion suppressor. The bandwidth as a function of center-of-mass energy is shown in Figure 2. The bandpass of the system is judged to be sufficient at all energies. The previous design called for using a larger bend angle in the CCX and CCY to reduce the necessary sextupole strength, which in turn would improve the bandpass; this is not considered necessary, given the bandpass as shown in Figure 2. The geometric luminosity (luminosity without disruption enhancement) as a function of energy is shown in Figure 3. Figure 3 includes effects of geometric and chromatic aberrations as well as synchrotron radiation in quads and bends.

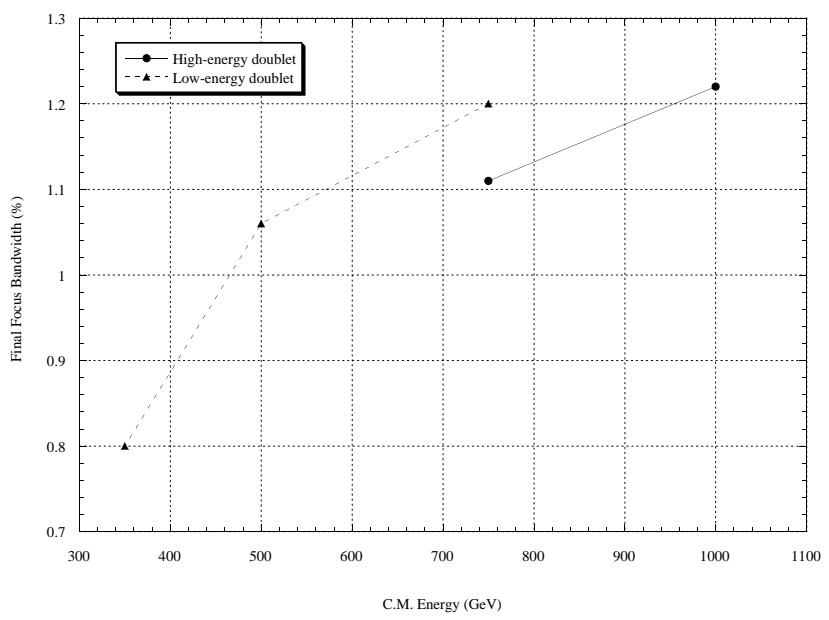

Figure 2: Luminosity bandwidth of NLC Final Focus as a function of center-of-mass energy.

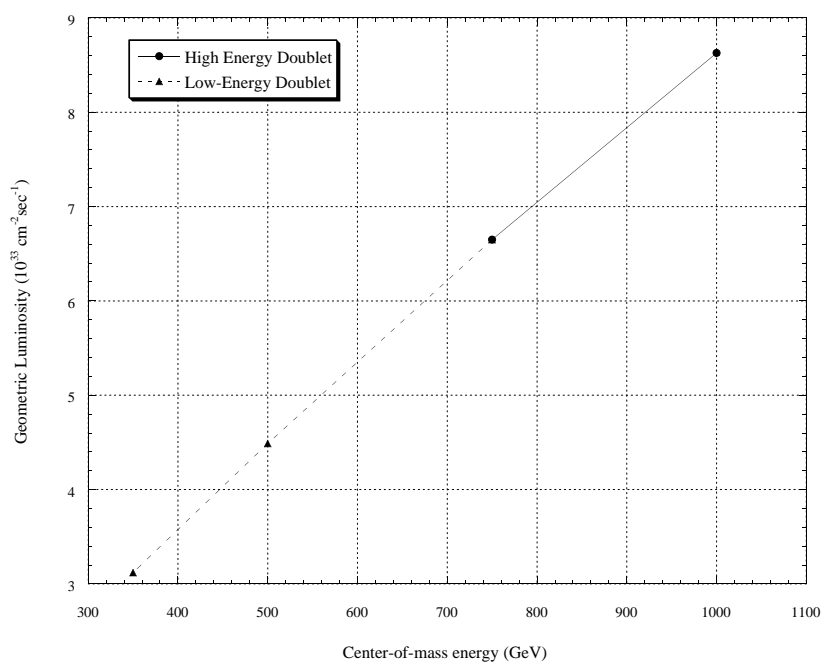

Figure 3: Luminosity of NLC Final focus as a function of center-of-mass energy.

\section{IR LAYOUT}

The NLC beams enter the detector with a 10 mrad horizontal angle with respect to the detector solenoid axis. The nonzero horizontal momentum in the solenoid causes a vertical displacement of the beams at the collision point. In addition, the beams have a very flat aspect ratio at the collision point, which can be severely impacted by coupling from the solenoid.

Previous studies of the interaction region have relied upon a "flux excluder", a solenoid around the last few quads which cancels the detector solenoid's effects. However, more complete modelling of the solenoid indicates that the effects of a real solenoid are much less severe than for a hard-edged field model: the fringe fields tend to cancel the effects of the main field. Thus the optical effects of the solenoid can be cancelled with very small skew quad fields and displacements of the last quad and the chromatic correction sextupoles at the level of a few microns. In addition, the solenoidal field falls off quickly with distance from the IP, and thus the permanent magnet quads ( 2 meters from the IP) are in no danger of demagnetizing due to the effect of the external solenoid field.

We conclude that the flux excluder can be removed from the IR design with only a miniscule impact on luminosity, and that no corrective elements are needed inboard of the last quadrupole magnet.

\section{FEEDBACKS AND TUNING SIMULATIONS}

Fast beam-based feedbacks were an essential component of the Stanford Linear Collider, and have been used extensively at synchrotron light sources to control instabilities and relax tolerances. Work has begun on identifying and defining the feedbacks needed in the beam delivery region of the NLC. These include several conventional feedbacks, which apply a correction to future bunch trains based on the measured performance of past train; dither feedbacks, which slowly vary a parameter (for example, IP dispersion) and measure a state (for example, luminosity) seeking an optimum value [3]; and a superfast IP feedback, which measures the beam-beam deflection of the first bunches of a train and applies a correction to subsequent bunches of the same train to achieve head-on collision.

\section{ACKNOWLEDGEMENTS}

The authors wish to thank R. Helm, L. Hendrickson, J. Irwin, and M. Woodley.

\section{REFERENCES}

[1] NLC ZDR Design Group, "Zeroth-Order Design Report for the Next Linear Collider,' SLAC Report 474 (1996).

[2] N.J. Walker et al, "Third-Order Corrections to the SLC Final Focus," Proc. PAC 9392 (1993).

[3] L. Hendrickson et al, "Luminosity Optimization Feedback in the SLC," SLAC-PUB-8027 (1999). 